\title{
Study of ionic surfactants interactions with carboxylated single-walled carbon nanotubes by using ion-selective electrodes
}

\author{
M. López-López a , E. Bernal ${ }^{\text {b }}$, M.L. Moyá ${ }^{\text {b }}$, F. Sanchez ${ }^{\text {b }}$, P. López-Cornejo ${ }^{\text {b,* }}$ \\ a Facultad de Ciencias Experimentales, Departamento de Ingeniería Química, Química Física y Ciencias de los Materiales, Universidad de Huelva, Campus 'El Carmen', E-21071, Spain \\ b Departamento de Química Física, Universidad de Sevilla, c/ Prof. García González 1, Sevilla 41012, Spain
}

\section{A R T I C L E I N F O}

\section{Article history:}

Received 31 January 2016

Received in revised form 10 March 2016

Accepted 10 March 2016

Available online 19 March 2016

\section{Keywords:}

Ion-selective electrodes

Carbon nanotubes

Surfactants

Interaction

Dispersion

Driving forces

\begin{abstract}
A B S T R A C T
Potentiometric measurements based on the use of ion-selective electrodes have been used to study the interaction (adsorption) of anionic and cationic surfactants with functionalized single-walled carbon nanotubes (SWCNT-COOH). According to results, the interaction is driven by hydrophobic forces between the hydrocarbon tails of the surfactants and the nanotube walls. Electrostatic interactions practically exert no influence on the interaction. Driving forces in the dispersion processes are different.
\end{abstract}

(c) 2016 Elsevier B.V. All rights reserved.

\section{Introduction}

Supramolecular chemistry, defined as the chemistry of molecular assemblies, studies the structures and functions of entities consisting of two or more species linked by noncovalent bonds. Electrostatic interactions, hydrogen bonds, $\pi-\pi$ stacking, metal-ligand, and hydrophobic interactions and stearic repulsions join in the formation of such assemblies [1-5]. Carbon nanotubes, CNT, are building blocks used in the construction of molecular assemblies. Due to their mechanical, optical, and electronic properties, they can be used in diverse fields. For instance, CNT have been used as sensors [6-8], or as vectors in gene transfection [9-10].

The walls of the carbon nanotubes are formed by curve sheets of graphene (a hexagonal lattice of carbon). There are two types of carbon nanotubes: the single-walled (SWCNT) and the multi-walled carbon nanotubes (MWCNT). All these structures show a low solubility in solution. Attractive van der Walls and $\pi-\pi$ stacking interactions provoke the assembly of the tubules into bundles or ropes. The separation (dispersion) of the carbon tubules can be improved by chemical methods. An example is the functionalization of the tubes via oxidation by strong acids like $\mathrm{H}_{2} \mathrm{SO}_{4}$ or $\mathrm{HNO}_{3}$. This results in the formation of carboxylic groups in the walls of nanotubes, which increases the charge in the sheets and decreases the agglomeration of the tubes. However, there are other procedures based on noncovalent interactions between

\footnotetext{
* Corresponding author.

E-mail address: pcornejo@us.es (P. López-Cornejo).
}

some chemical species and nanotubes. Surfactants are frequently used as dispersing agents. Their amphiphilic nature favors their adsorption on the tube walls and decreases the ability of the carbon nanotubes to agglomerate in bundles.

Several studies about the interactions between surfactants and carbon nanotubes have been carried out [11-14]. However, the mechanism of such interaction is not clear. Indeed, different authors have investigated the influence of the polar head group charge on the dispersion of the nanotubes, but no clear conclusions were reached $[15,16]$. Inconclusive results were also obtained when sodium dodecyl sulfate (SDS) was used as dispersing agent. While some authors propose that cylindrical micelles are formed on the nanotubes [17], others think that hemi-micelles are adsorbed on the nanotube walls [18]. However, following the suggestion of Matarredona et al. [19] pointing out that the hemi-micellar adsorption of surfactants on single carbon nanotubes is sterically and energetically unfavorable, Yurekli et al. [20] suggested that a structureless random adsorption, with no preferential arrangement of the head or tail, takes place.

In order to shed light on the surfactant/carbon nanotube interactions, quantitative information about the interactions (or adsorption) of some commercial cationic and anionic surfactants with functionalized carbon nanotubes (SWCNT-COOH) was obtained. Taking advantage of the known benefits offered by potentiometric techniques (sensitivity, precision, reproducibility, selectivity, and low cost among others), the study was carried out by using electromotive forces measurements. For that, ion-selective electrodes for ionic surfactants were prepared. 
$\mathrm{Ag} / \mathrm{AgCl} \mid$ reference solution || selective membrane || test solution $\mid \mathrm{Ag} / \mathrm{AgCl}$

$$
E=E^{O^{\prime}}-2.303 \frac{R T}{z_{i} F} \log a_{i}
$$

Scheme 1.

\section{Experimental}

\subsection{Reactants}

The surfactants investigated were hexadecyltrimethylammonium bromide (CTAB), dodecyltrimethylammonium bromide (DoTAB), octyltrimethylammonium bromide (OTAB), and sodium dodecyl sulfate (SDS); all of them received from Sigma Aldrich (R grade). The all surfactant concentrations used were always lower than the CMC. A buffer phosphate solution at $\mathrm{pH} 9$ was used to avoid the protonation of the carboxylic groups [21]. In order to study the influence of the SWCNT charge, some measurements were done at $\mathrm{pH} 7$ (phosphate) and 1.5 $(\mathrm{HCl})$. The functionalized carbon nanotubes were from NanoLab Inc.

\subsection{Potentiometric measurements}

Electromotive forces of solutions containing surfactant and SWCNT$\mathrm{COOH}$ were measured by using selective electrodes of the different surfactants previously prepared by us.

Selective membrane electrodes of the cationic (CTAB, DoTAB, and OTAB) and anionic (SDS) surfactants used in this work were synthesized as follows:

1) First, ion pairs of the cationic and ionic surfactants were prepared. Thus, in the case of the cationic surfactants, ion pairs were prepared in water by mixing equimolecular amounts of the cationic surfactants and sodium tetraphenylborate. The ion pair of the anionic surfactant was obtained mixing in water equimolecular amounts of SDS and CTAB. All mixtures resulted in white precipitates, which were repeatedly washed with water and recrystallized with tetrahydrofu$\operatorname{ran}(\mathrm{THF})$.

2) Membranes were prepared mixing PVC (0.35 g), bis(2ethylhexil)phthalate $(1.15 \mathrm{~g})$, and the corresponding ion pair $(10 \mathrm{mg}$ ) and dissolving the mixture in $6 \mathrm{ml}$ of THF with continuous stirring. Obtained the solution, the solvent was evaporated on air.

3) The membrane was placed at the end of a PVC tube, using PVC paste and THF as glue. The resulting membrane electrode was conditioned

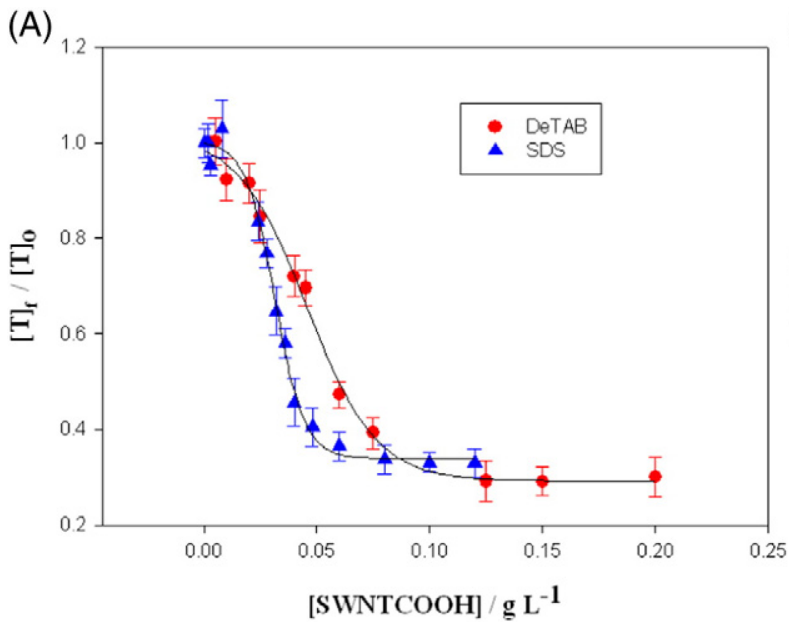

for various hours in a reference solution $\left(\mathrm{Na}_{2} \mathrm{HPO}_{4} 0.01 \mathrm{M} / \mathrm{NaCl}\right.$ $0.02 \mathrm{M}, \mathrm{pH} 9$ ) containing the corresponding surfactant.

Scheme 1 shows a picture of the cell used in the electromotive force measurements and the corresponding Nernst equation (where $a_{i}$ is the activity of the ionic surfactants).

The surfactant concentrations of the reference solutions used were $1 \times 10^{-3} \mathrm{~mol} \mathrm{dm}^{-3}$ for SDS, $2 \times 10^{-2} \mathrm{~mol} \mathrm{dm}^{-3}$ for OTAB, $2 \times 10^{-3} \mathrm{~mol} \mathrm{dm}^{-3}$ for DoTAB, and $1 \times 10^{-4} \mathrm{~mol} \mathrm{dm}^{-3}$ for CTAB. A salt bridge of $\mathrm{KCl}$ in agar gel was used in all the measurements. Electromotive forces were measured in a custom-built electrometric amplifier using a INA 116 ultra-low input bias current instrumentation amplifier, followed by a unity-gain Sallen-Key low-pass filter $[n=4$, $\left.f_{\mathrm{c}}=15 \mathrm{~Hz}(-3 \mathrm{db})\right]$. The response was monitored using a Keithley 2110 51ํㄹㄹ Digital Multimeter, which was interfaced to a PC through a USB interface with an accuracy of $\pm 0.1 \mathrm{mV}$. In all cases, the membrane gave a Nernstian response to the logarithm of the surfactant concentration with slope values close to $59 \mathrm{mV}$ at $298.1 \pm 0.1 \mathrm{~K}$. The temperature was maintained constant by using a glass cell with a thermostated water bath. The concentration of the free surfactant was estimated using a calibration curve for each selective electrode.

\subsection{Transmission electronic microscopy (TEM)}

Transmission electronic microscopy samples were prepared as follows: a drop of the aqueous solution containing surfactant and/or SWCNT-COOH was deposited on a cooper grid coated with a carbon film. The grid was dried to air at room temperature.

The pictures were visualized with a Philips CM200 electron microscope. The concentrations used were [SWCNT-COOH] $=0.05 \mathrm{~g} / \mathrm{l}$ $[$ DeTAB $]=5 \times 10^{-5} \mathrm{~mol} \mathrm{dm}^{-3}$ and $[$ SDS $]=5 \times 10^{-5} \mathrm{~mol} \mathrm{dm}^{-3}$. All the concentrations were prepared in buffer phosphate at $\mathrm{pH} 9$ $\left(\mathrm{Na}_{2} \mathrm{HPO}_{4} 0.01 \mathrm{M} / \mathrm{NaCl} 0.02 \mathrm{M}\right)$.

\section{Results and discussion}

As can be seen in Fig. 1, electromotive force measurements gave a decreasing of the free surfactant concentration when the SWCNT-

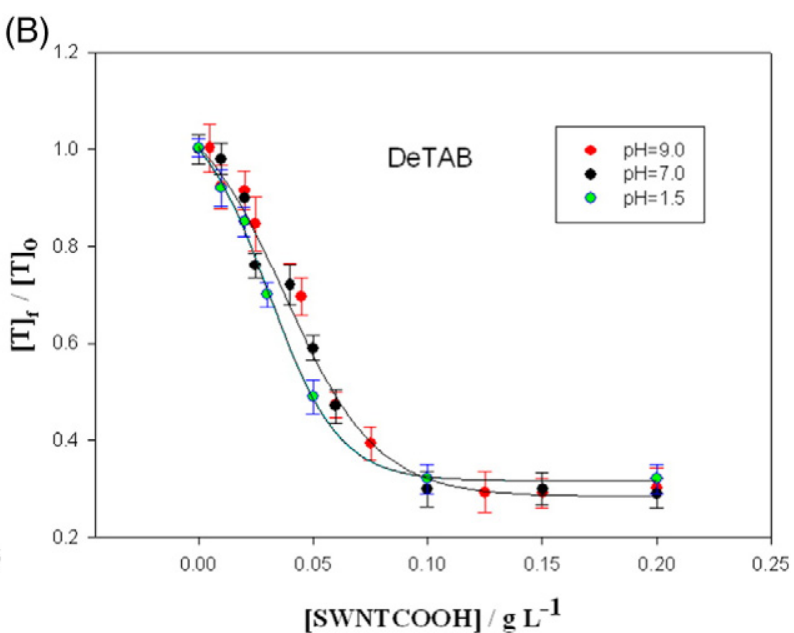

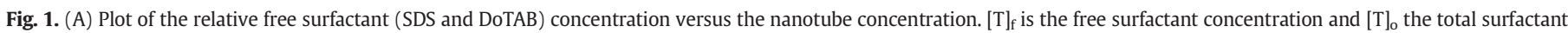
concentration added to the solution. (B) Relative free DoTAB concentration at different [SWCNT-COOH] and different pH. Data represent mean \pm SD $(n=4)$. 


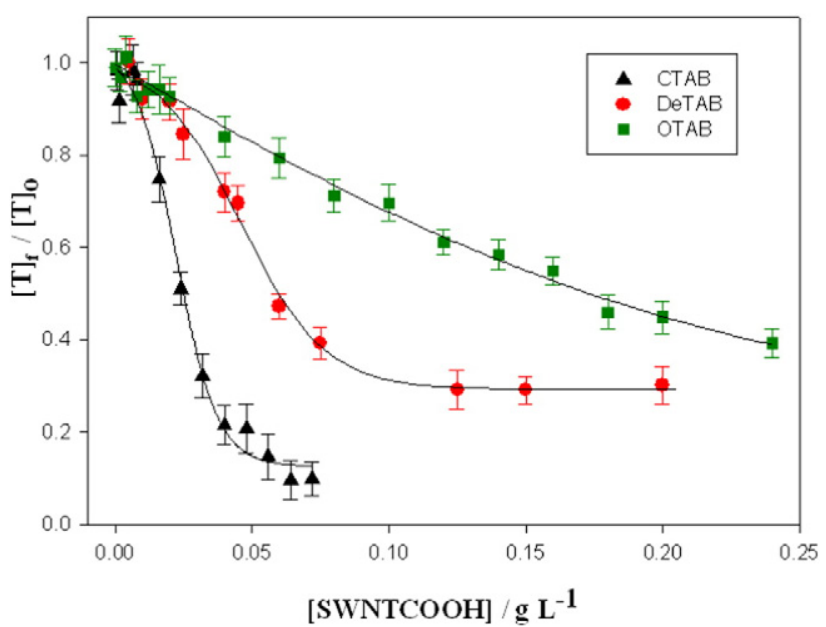

Fig. 2. Plot of the relative free cationic surfactant (CTAB, DoTAB, and OTAB) concentration versus the nanotube concentration. Data represent mean $\pm \operatorname{SD}(n=4)$.

$\mathrm{COOH}$ concentration increases. The free surfactant concentration shows a sigmoidal dependence on the nanotube concentration. This trend indicates a cooperative character of the interaction (the interactions of a ligand with a receptor are cooperative when the interaction of a first ligand to the receptor makes the interaction of a second ligand to the same receptor stronger and so on). Similar behaviors are obtained for SDS and DoTAB (see Fig. 1A). The lack of influence of the surfactant headgroup charge on the surfactant adsorption to the SWCNT suggests random interactions, where the electrostatic contribution does not play an important role. The hydrophobic interactions between the hydrocarbon tails of the surfactant and the carbon nanotubes would be the driving force in the adsorption mechanism. The ionization degree of the carboxylated CNT, which can be varied with the $\mathrm{pH}$ of the medium, practically exerts no influence on the free surfactant concentration (see Fig. 1B). To go further in our study, the interaction of three cationic surfactants with the same hydrophilic headgroup and different tail lengths with SWCNT-COOH were investigated. Fig. 2 shows the results obtained from electromotive force measurements. As can be seen, the free surfactant concentration diminishes upon increasing the SWCNT$\mathrm{COOH}$ concentration. This decrease is stronger with a larger length of the hydrophobic tail $(C T A B>$ DoTAB $>$ OTAB $)$.
It is well accepted in the literature that SDS is the best and the most used surfactant for the dispersion of nanotubes [22-24]. At first, according to our results, SDS and DoTAB could be indistinctly used in the dispersion of SWCNT-COOH. However, it is important to emphasize the difference between interaction/adsorption and dispersion. It is easy to find in the literature papers in which both terms are used when talking about finding the best dispersant of nanotubes. However, a surfactant that strongly interacts with the nanotubes has not to be a good dispersing agent. Indeed, the surfactant headgroup plays an important role in the dispersion process, but not in the adsorption process. In order to clarify this point, the results obtained in this work will be considered. SDS is an anionic surfactant whose hydrophobic tail is adsorbed on the nanotube walls. In the present work, at pH 9 or 7, the carboxylic groups of the SWCNT-COOH will be mostly dissociated [21], and the functionalized graphene sheets are expected to be negatively charged. The adsorption of SDS molecules to the negatively charged nanotubes will increase their negative charge, and the dispersion of the nanotubes will be favored since the electrostatic repulsions among tubes increases (see Fig. $3 \mathrm{~A}$ ). However, the adsorption of cationic surfactants (CTAB, DoTAB, and OTAB) on the functionalized nanotubes results in a diminution of their negative charge, diminishing the electrostatic repulsions among tubes and making their dispersion more difficult. Therefore, the cationic surfactants are expected to be worse dispersing agents than anionic surfactants for the SWCNT-COOH investigated (see Fig. 3B). This assumption has been confirmed by transmission electron microscopy and by UV-vis spectroscopy. Fig. 4A-F show the dispersion of SWCNT-COOH in the absence and presence of SDS and DoTAB with the same magnification. It is clear that a poorest dispersion is observed in the absence of any surfactant. The presence of SDS causes a better dispersion than that of DoTAB. Individual nanotubes (but not bundled CNTs) absorb in the UV-vis region [25]. Bearing this in mind, UV-vis spectroscopy measurements of surfactant-SWCNT solutions were run. Results, collected in Fig. 4G and H, demonstrate a higher absorbance (and a higher individual CNTs concentration therefore) for SDS than for DoTAB at $\mathrm{pH} 7$, and higher at $\mathrm{pH} 1.5$ than at $\mathrm{pH} 7$ for the DoTABSWCNT system. This confirms our conclusions.

It must be noted that this behavior in the interaction (adsorption) and dispersion of CNTs with ionic surfactants occurs with both charged and uncharged graphene sheets, as results obtained at different $\mathrm{pH}$ have demonstrated.
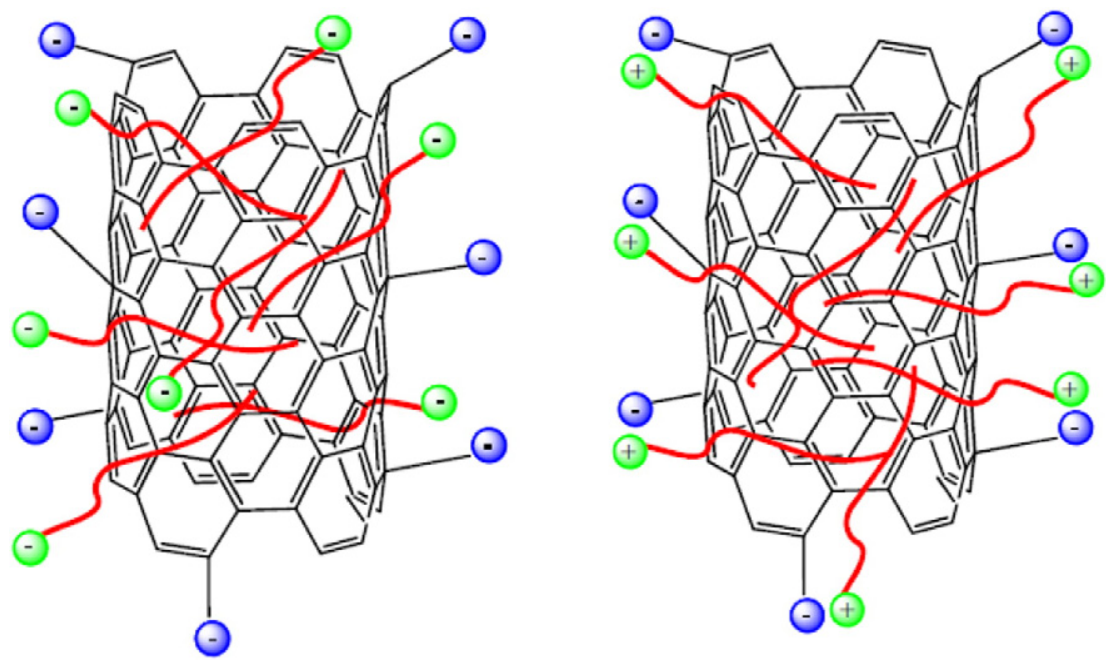

Fig. 3. Schematic picture of the interaction (adsorption) of the anionic (A) and cationic (B) surfactant on the SWCNT-COOH wall. 

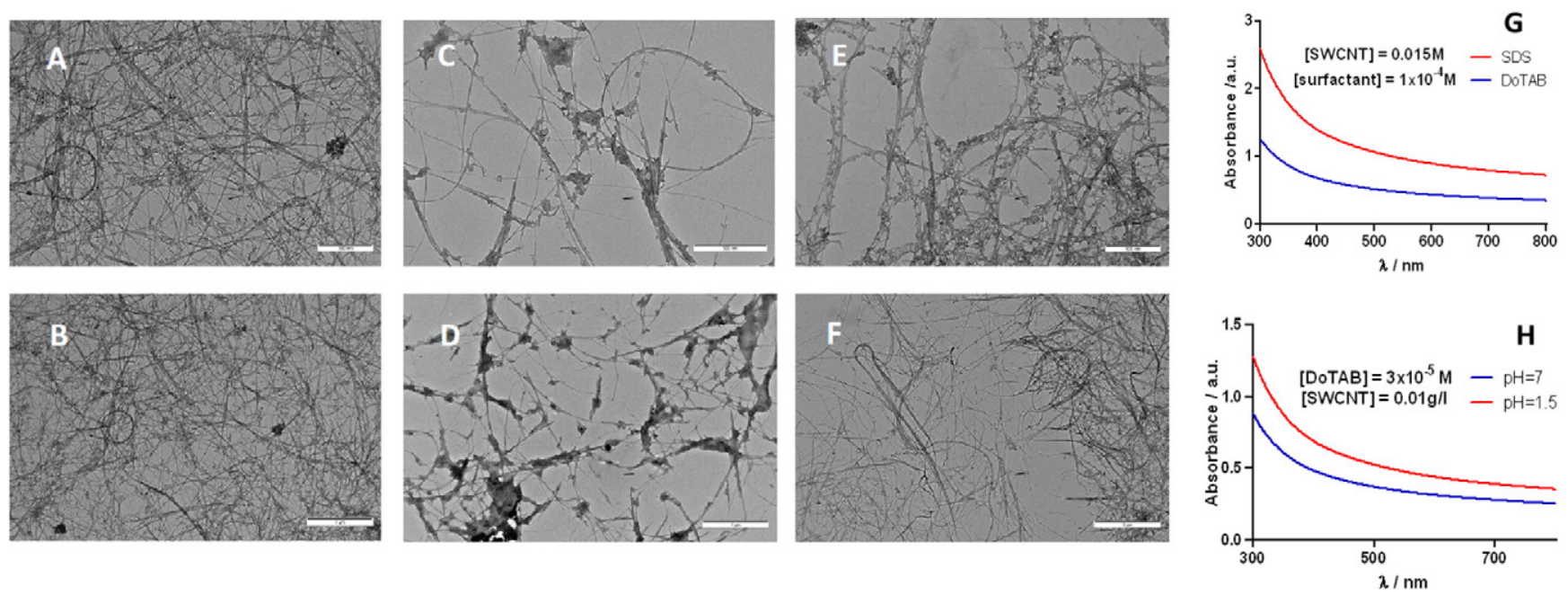

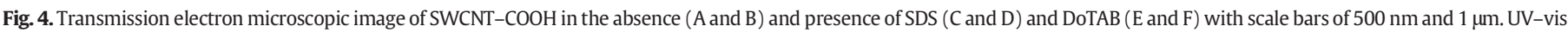
spectra of SDS/SWCNT and DoTAB/SWCNT at pH $7(G)$ and of DoTAB/SWCNT at different pH values $(H)$.

\section{Conclusions}

In summary, the tendency of a surfactant to interact (adsorb) with SWCNT-COOH and its quality as dispersing agent do not have to coincide. The hydrophobic interaction contribution is the driving force in the adsorption process, while electrostatic interactions (together hydrophobic forces) play a major role in the dispersion process. In general, the nature of the carbon nanotubes, as well as the solvent, must also be considered. It must be noted that, to the authors' knowledge, it is the first time that quantitative information about the surfactant/carbon nanotubes interactions are provided. The importance of the use of potentiometric measurements to get information about nanosystems such as carbon nanotubes is also noteworthy.

\section{Conflict of interest}

Authors declare no competing financial interest.

\section{Acknowledgments}

This work was supported by the Consejería de Educación y Ciencia de la Junta de Andalucía (Proyecto de Excelencia P12-FQM-1105) and the European Union (Feder Funds).

\section{References}

[1] Y. Liu, D.-S. Guo, E.-C. Yang, H.-Y. Zhang, Y.-L. Zhao, The structures and thermodynamics of complexes between water-soluble calix[4]arenes and dipyridinium ions, Eur. J. Org. Chem. 162-170 (2005)

[2] L.-H. Wang, D.-S. Guo, Y. Chen, Y. Liu, Thermodynamics of interactions between organic ammonium ions and sulfonatocalixarenes, Thermochim. Acta 443 (2006) 132-135.

[3] Y. Liu, D.-S. Guo, H.-Y. Zhang, Y.-H. Ma, E.-C. Yang, The structure and thermodynamics of calix[n]arene complexes with dipyridines and phenanthroline in aqueous solution studied by microcalorimetry and NMR spectroscopy, J. Phys. Chem. B 110 (2006) 3428-3434

[4] P. Lopez-Cornejo, B. Bote, R. Felix, I. Infantes, P. Lopez, A. Martin, E. Mateos, M. Perez, A. Rojas, R. Suarez, Binding of $\mathrm{Ru}\left(\mathrm{NH}_{3}\right)_{5} \mathrm{pz}^{2+}$ to 4-sulfocalix[4]arene sodium salt. Effects of the host - guest interaction on electron transfer processes, J. Phys. Chem. B 113 (2009) 12721-12726.

[5] J.-M. Lehn, Perspectives in chemistry-steps towards complex matter, Angew. Chem. Int. Ed. 52 (2013) 2836-2850.

[6] J. Chen, T.O. Tran, M.T. Ray, D.B. Brunski, J.C. Keay, D. Hickey, M.B. Johnson, D.T. Glatzhofer, D.W. Schmidtke, Effect of surfactant type and redox polymer type on single-walled carbon nanotube modified electrodes, Langmuir 29 (2013) 10586-10595.

[7] K. Yum, J.H. Ahn, T.P. McNicholas, P.W. Barone, B. Mu, J.H. Kim, R.M. Jain, M.S. Strano, Boronic acid library for selective, reversible near-infrared fluorescence quenching of surfactant suspended single-walled carbon nanotubes in response to glucose, ACS Nano 6 (2011) 819-830.

[8] K. Yum, T.P. McNicholas, B. Mu, M.S. Strano, Single-walled carbon nanotube-based near-infrared optical glucose sensors toward in vivo continuous glucose monitoring, J. Diabetes Sci. Technol. 7 (2013) 72-87.

[9] A. Bianco, K. Kostarelos, M. Prato, Applications of carbon nanotubes in drug delivery Curr. Opin. Chem. Biol. 9 (2005) 674-679.

[10] L. Shi, D. Shi, M.U. Nollert, D.E. Resasco, A. Striolo, Single-walled carbon nanotubes do not pierce aqueous phospholipid bilayers at low salt concentration, J. Phys. Chem. B 117 (2013) 6749-6758.

[11] L. Vaisman, H.D. Wagner, G. Marom, The role of surfactants in dispersion of carbon nanotubes, Adv. Colloid Interf. Sci. 128-130 (2006) 37-46.

[12] F. Tardini, C. La Mesa, Attempts to control depletion in the surfactant-assisted stabilization of single-walled carbon nanotubes, Colloids Surf. A Physicochem. Eng. Asp. 443 (2014) 123-128.

[13] Y. Chen, R.N. Gunasinghe, X.-Q. Wang, Y. Pang, Selective dispersion of single-walled carbon nanotubes by a cationic surfactant, RSC Adv. 3 (2013) 25097-25102.

[14] B. Sohrabi, N. Poorgholami-Bejarpasi, N. Nayeri, Dispersion of carbon nanotubes using mixed surfactants: experimental and molecular dynamics simulation studies, J. Phys. Chem. B 118 (2014) 3094-3103.

[15] T. Chatterjee, K. Yurekli, V.G. Hadjiev, R. Krishnamootri, Single-walled carbon nanotube dispersions in poly (ethylene oxide), Adv. Funct. Mater. 15 (2005) 1832-1838.

[16] L. Vaisman, G. Marom, H.D. Wagner, Dispersions of surface-modified carbon nanotubes in water-soluble and water-insoluble polymers, Adv. Funct. Mater. 16 (2006) 357-363.

[17] M.J. O'Connell, S.M. Bachilo, C.B. Huffman, V.C. Moore, M.S. Strano, et al., Band gap fluorescence from individual single-walled carbon nanotubes, Science 297 (2002) 593-596.

[18] M. Mackiewicz, G. Surendran, H. Remita, B. Keita, G. Zhang, et al., Supramolecular self-assembly of amphiphiles on carbon nanotubes: a versatile strategy for the construction of CNT/metal nanohybrids, application to electrocatalysis, J. Am. Chem. Soc. 130 (2008) 8110-8111.

[19] O. Matarredona, H. Rhoads, Z.R. Li, J.H. Harwell, L. Balzano, R.E. Resasco, Dispersion of single-walled carbon nanotubes in aqueous solutions of the anionic surfactant NaDDBS, J. Phys. Chem. B 107 (2003) 13357-13367.

[20] K. Yurekli, C.A. Mitchell, R. Krishnamoorti, Small-angle neutron scattering from surfactant-assisted aqueous dispersions of carbon nanotubes, J. Am. Chem. Soc. 126 (2004) 9902-9903.

[21] H. Hu, Y. Ni, V. Montana, R.C. Haddon, V. Parpura, Chemically functionalized carbon nanotubes as substrates for neuronal growth, Nano Lett. 4 (2004) 507-511.

[22] A.J. Blanch, J.G. Shapter, Surfactant concentration dependent spectral effects of oxygen and depletion interactions in sodium dodecyl sulfate dispersions of carbon nanotubes, J. Phys. Chem. B 118 (2014) 6288-6296.

[23] W.H. Duan, Q. Wang, F. Collins, Dispersion of carbon nanotubes with SDS surfactants: a study from a binding energy perspective, Chem. Sci. 2 (2011) 1407-1413.

[24] R. Rastogi, R. Kaushal, S.K. Tripathi, A.L. Sharma, I. Kaur, L.M. Bharadwaja, Comparative study of carbon nanotube dispersion using surfactants, J. Colloid Interface Sci. 328 (2008) 421-428.

[25] J. Yu, N. Grossiord, C.E. Koning, J. Loos, Controlling the dispersion of multi-wall carbon nanotubes in aqueous surfactant solution, Carbon 45 (2007) 618-623. 